\title{
REMOTE CONTEXTUAL FEAR RETRIEVAL ENGAGES ACTIVITY FROM SALIENCE NETWORK REGIONS IN RATS
}

Short title: Remote fear memory engages the salience network in rats

Moisés dos Santos Corrêa ${ }^{1}$, Gabriel David Vieira Grisanti ${ }^{1}$, Isabelle Anjos Fernandes Franciscatto ${ }^{1}$, Tatiana Suemi Anglas Tarumoto이 ${ }^{1}$ Paula Ayako Tiba ${ }^{1}$, Tatiana Lima Ferreira ${ }^{1}$, Raquel Vecchio Fornari $^{1}$

(1) Center for Mathematics, Computing and Cognition (CMCC), Universidade Federal do ABC (UFABC), São Bernardo do Campo, SP, Brazil

\section{Authors' e-mails and ORCID:}

Moisés dos Santos Corrêa (first author): mscorrea.86@gmail.com - Orcid: 0000-0002-4488-0922

Gabriel David Vieira Grisanti: gabrielgrisanti96@gmail.com - Orcid: 0000-0002-2515-1943

Isabelle Anjos Fernandes Franciscatto: isabelle.anjos.f@outlook.com - Orcid: 0000-0001-8653-2726

Tatiana Suemi Anglas Tarumoto: tatitarumoto@gmail.com - Orcid: 0000-0002-0557-3305

Paula A. Tiba: paula.tiba@ufabc.edu.br - Orcid: 0000-0001-8030-9232

Tatiana L. Ferreira: tatiana.ferreira@ufabc.edu.br - 0000-0002-2805-513X

Raquel V. Fornari (correspondence): raquel.fornari@ufabc.edu.br - Orcid: 0000-0003-1330-6263

\section{Color version only on online version, in print black and white}


Dos Santos Corrêa et al, 2021

\begin{abstract}
The ability to retrieve contextual fear memories depends on the coordinated activation of a brain-wide circuitry. Transition from recent to remote memories seems to involve the reorganization of this circuitry, a process called systems consolidation that has been associated with time-dependent fear generalization. However, it is not known whether emotional memories acquired under different levels of stress can undergo different systems consolidation processes. Here, we explored the activation pattern and functional connectivity of key brain regions associated with contextual fear conditioning (CFC) retrieval after recent ( 2 days) or remote (28 days) memory tests performed in rats submitted to strong (1.0mA footshock) or mild ( $0.3 \mathrm{~mA}$ footshock) training. We used brain tissue from Wistar rats from a previous study, where we observed that increasing training intensity promotes fear memory generalization over time, possibly due to an increase in corticosterone levels during memory consolidation. Analysis of Fos expression across 8 regions of interest (ROIs) allowed us to identify coactivation between them at both timepoints following memory recall. Our results showed that strong CFC elicits higher Fos activation in the anterior insular and prelimbic cortices during remote retrieval, which was - along with the basolateral amygdala - positively correlated with freezing. Rats trained either with mild or strong CFC showed a broad functional connectivity at the recent timepoint whereas only animals submitted to the strong CFC showed a widespread loss of coactivation during remote retrieval. Our findings suggest that increasing training intensity results in differential processes of systems consolidation, possibly associated with increased post-training corticosterone release, and that strong CFC engages activity from areas associated with the salience network during remote retrieval.
\end{abstract}

KEYWORDS: systems consolidation; stress; basolateral amygdala; anterior insular cortex; prelimbic cortex 
Dos Santos Corrêa et al, 2021

\section{INTRODUCTION}

Traumatic events may induce pathological forms of fear memory, including overgeneralized fear, which contribute to symptoms of stress and anxiety-related disorders together with posttraumatic stress disorder (PTSD) (Mahan and Ressler, 2012). Recent advances suggest that long-term contextual fear memory retrieval seems to be dependent on the coordinated activation of a brain-wide engram circuit (Wheeler et al., 2013). Furthermore, the transition of a recent to remote memory is thought to involve a reorganization of this circuit, a process called systems consolidation (DeNardo et al., 2019; Moscovitch et al., 2016). Traditionally, recent contextual fear conditioning (CFC) retrieval is associated with high contextual specificity and neuronal activity in the hippocampal formation, especially its dorsal portion (dHPC). On the other hand, activity in neocortical regions have been associated with generalized, remote contextual memories (Frankland et al., 2004; Winocur et al., 2010). Nonetheless, evidence also suggests that the dHPC may be engaged in remote retrieval (Barry and Maguire, 2019; Goshen et al., 2011; Tanaka et al., 2014), whereas the medial pre-frontal cortex (mPFC) may also be involved in the retrieval of precise remote memories (Matos et al., 2019; Moscarello and Maren, 2018). Hence, the mechanisms by which the temporal reorganization of the engram circuit occurs is still controversial.

Parallel to the research into systems consolidation and time-dependent fear generalization, other studies also suggest that recognition, spatial or emotional memory engram cells are allocated during acquisition in several brain regions other than the hippocampus (Roy et al., 2016). Some of these regions are the amygdala (Redondo et al., 2014; Wang et al., 2009), the anterior insular cortex (aIC) (Sano et al., 2014) and the mPFC, especially the prelimbic cortex (PL) (Zhang et al., 2011). These regions are part of the so-called Salience Network (SN) and are highly responsive to arousing situations in humans (Hermans et al., 2014b) and in mice (Mandino et al., 2021; Zerbi et al., 2019). Strong, aversive events are thought to prompt a shift of memory encoding dependence from a hippocampal-dominated engram circuit to $\mathrm{SN}$ regions during memory acquisition and consolidation, favoring the encoding of emotional memories (Arnsten, 2009; Hermans et al., 2014b). In contrast, there is evidence that CFC in rats is associated with decreased functional connectivity between the retrosplenial cortex (RSC) - a region considered part of the "Default-mode-like network" (DMN) in rodents - to the aIC (Ji et al., 2018; Upadhyay et al., 2011). Furthermore, a comprehensive neuroimaging study using human data showed that generalized fear is associated with decreased activation in nodes of the default mode network, especially the HPC, and increased activation of the aIC, mPFC and ACC (Webler et al., 2021). Hence, this shift in memory systems resultant from higher stress and engagement of different neurocognitive networks elicited by strong aversive situations may trigger different processes of systems consolidation. 
Several studies have also linked increasing levels of arousal and stress hormones with strengthened contextual fear memories and to an acceleration of the rate of time-dependent fear generalization/systems consolidation (Dos Santos Corrêa et al., 2021, 2019; Pedraza et al., 2016). More recently, studies showed that post-training activation of the noradrenergic and glucocorticoid systems modulates contextual fear memory specificity (Atucha et al., 2017; Dos Santos Corrêa et al., 2021; Gazarini et al., 2021; Roozendaal and Mirone, 2020). Therefore, it is possible that interfering with arousal levels by changing training parameters may affect contextual memory accuracy and the process of time-dependent fear generalization/systems consolidation through changes in the engagement of neurocognitive networks (Schwabe, 2017). To explore this question, a recent study from our laboratory investigated the rate of time-dependent fear memory generalization following increasing intensities of CFC training. Our data showed that mild-footshocks $(0.3 \mathrm{~mA})$ elicited lower corticosterone (CORT) levels and accurate remote contextual memories while, on the other hand, strong footshocks $(1.0 \mathrm{~mA})$ increased CORT release during memory consolidation, which was associated with remote generalized fear (Dos Santos Corrêa et al., 2019). These results indicate that increasing levels of arousal elicited different appraisal of the aversive experience, promoting fear memory generalization possibly due to an increase in glucocorticoid activity during memory consolidation.

In the present study we examined the expression of the immediate early gene product Fos following memory retrieval in the same rats used in the previous study (Dos Santos Corrêa et al., 2019). Here, we explored whether there were changes in functional connectivity of key brain regions associated with the SN, the DMN-like or both networks (Mandino et al., 2021; Zerbi et al., 2019). We hypothesized that increasing footshock intensity during $\mathrm{CFC}$ training would elicit different processes of systems consolidation, which can be observed through changes in functional connectivity between these regions.

\section{METHODS}

\section{Subjects and behavioral task}

For the present study we used a subset of animals from a previously published experiment

3 (Dos Santos Corrêa et al., 2019) and the behavioral data from these animals was extracted from the 4 previously published dataset ([dataset] dos Santos Corrêa et al., 2019). These animals were three5 month old male Wistar rats, obtained from Instituto Nacional de Farmacologia (INFAR-UNIFESP 6 (total $\mathrm{n}=42$; weighing between $275-385 \mathrm{~g}$ at time of training). All rats were kept in controlled 7 conditions of temperature $\left(23 \pm 2^{\circ} \mathrm{C}\right)$ and maintained on a $12 \mathrm{~h} \mathrm{light/dark}$ cycle with free access to 8 food and water. Behavioral experiments were conducted during the light phase of the cycle, from 11 9 am to $4 \mathrm{pm}$, during the rat's nadir of corticosterone circadian rhythm. All procedures were conducted 
according to the guidelines and standards of CONCEA - Conselho Nacional de Controle de Experimentação Animal (Brazilian Council of Animal Experimentation) and were previously approved by the Ethics Committee on Animal Use - UFABC (CEUA - protocol numbers 5676291015 and 7479070916)

Contextual fear conditioning experiments were conducted as described before (Dos Santos Corrêa et al., 2019) in a windowless room containing the conditioning chamber (32 cm wide, $25 \mathrm{~cm}$ high and $25 \mathrm{~cm}$ deep, Med-Associates VFC-008). Rat freezing behavior was monitored via a frontal near infra-red-light camera. Freezing was assessed using an automated scoring system (Video Freeze, Version 1.12.0.0, Med-Associates), which digitized the video signal at $30 \mathrm{~Hz}$ and compared frame by frame movement to determine the amount of time spent freezing. The context was characterized by a grid floor composed of 20 stainless steel rods (diameter: $4.8 \mathrm{~mm}$ ), top and front walls made of transparent polycarbonate, a back wall made of white acrylic, stainless-steel sidewalls and a drop pan below the floor grid. The light in the conditioning box remained on, and a background noise was emitted during the training and test sessions. The chamber was cleaned with alcohol $10 \%$ before and after each rat.

During training, rats were placed in the conditioning chamber for four minutes. After two minutes they were presented with three unsignalled footshocks $(1 \mathrm{~s}$ duration, 0.3 or $1.0 \mathrm{~mA}, 30$ seconds apart). Following the last footshock rats remained in the chamber for another minute, and then were returned to their home cage. Control rats underwent the same training procedure but did not receive the footshocks during the conditioning session (Context Only-C.O.). After each training condition, separate groups of rats were tested either 2 days (C.O. $\mid$ Recent, $n=5 ; 0.3 \mathrm{~mA} \mid$ Recent, $n=6$; $1.0 \mathrm{~mA} \mid$ Recent, $\mathrm{n}=6$ ) or 28 days (C.O.|Remote, $\mathrm{n}=5 ; 0.3 \mathrm{~mA} \mid$ Remote, $\mathrm{n}=6,1.0 \mathrm{~mA} \mid$ Remote, $\mathrm{n}=6$ ) later. During testing, rats were placed back in the training context for five minutes and freezing time was assessed. No footshock was delivered during test sessions. Another control group of rats was only handled but did not undergo CFC training or testing (Homecage, Recent, $n=4$, Remote, $n=4$ ).

\section{Perfusion and histology}

Ninety minutes following the end of the test session, rats were deeply anesthetized with $30 \%$ urethane and then perfused transcardially with $100 \mathrm{~mL}$ saline followed by $500 \mathrm{~mL}$ of ice-cold $4 \%$ paraformaldehyde (PFA) dissolved in phosphate buffer saline. The brains were removed, fixed for at least 2 hours in PFA, then transferred to $30 \%$ sucrose solution and stored at $4^{\circ} \mathrm{C}$. After dehydration, these brains were frozen in isopentane on dry ice and stored at $-80{ }^{\circ} \mathrm{C}$. Frozen coronal sections $(40$ $\mu \mathrm{m})$ were cut in a microtome with dry ice and stored in 5 serial sets. One set was collected in glass slides and Nissl-stained to cytoarchitectural delineation of brain areas, and another set was used for Fos immunolabeling. 
Dos Santos Corrêa et al, 2021

\section{$\underline{3 \text { Immunohistochemistry and cell counting }}$}

Free-floating sections underwent antigen retrieval (10mM Sodium Citrate, ph 8.5) for 30 minutes at $80{ }^{\circ} \mathrm{C}$, and peroxidase blocking $(0.3 \%$ hydrogen peroxide in $0.02 \mathrm{M}$ PBS) followed by indirect antibody staining protocols aiming Fos labeling by avidin-biotin reaction. Sections were incubated at $4{ }^{\circ} \mathrm{C}$ for 72 hours with rabbit anti-cfos polyclonal antibody $(1: 10,000$ AbCam) in $2 \%$ normal goat serum, $0.3 \%$ Triton X-100 and 0.02M PBS. After washes, incubation with secondary antibody (anti-rabbit Ab from goat diluted in 1:200 - BA1000, Vector, in 0.02M PBS and 0.3\% Triton X-100) took 90 minutes in room temperature. The secondary antibody was biotinylated in the avidin-biotin-complex solution (1:200, ABC Kit, VectaStain Elite, Vector) in 0.02M PBS and 0.3\% Triton X-100. The peroxidase complex was visualized using the chromogen diaminobenzidine 3,3tetrachloride (DAB Kit, Vector). Finally, sections were mounted on gel-coated slides and left to dry for at least $48 \mathrm{~h}$. After drying, sections were diaphanized, coverslipped with DPX mountant medium (Sigma 06522) and left to dry for at least a week.

Fos expression was analyzed in 8 regions of interest (ROIs, Table 1). Images from each ROI were acquired using either a Zeiss microscope model Axio image 2 at 20x magnification or a Leica microscope model DM5500 at 10x magnification, following the same frame size, image size and area for each microscope. One ROI (anterior retrosplenial cortex) was smaller than the standard area and was delimited using the ImageJ software (NIH, Washington, United States). The anatomical delimitation of each ROI was based on Paxinos and Watson rat brain atlas (Paxinos and Watson, 2007) and on adjacent Nissl-stained sections. Table 1 shows the information regarding anteroposterior coordinates of slices, image size, amplification and number of bilateral sections for each ROI. Image $\mathrm{B} \square$ (1.52a) was used to count cells automatically following parameters of size, circular shape and contrast level adapted to each image but within a pre-established range to ensure consistency. Experimenters were blinded to the experimental group. Cell density of each photo was obtained dividing the number of cells to the total area of the image. Mean Fos density of the photos from each animal, either for recent or remote timepoints, was normalized by the averaged Fos density of homecage rats that were euthanized in the same days of the other groups. The experimental unit was the cell Fos density normalized by the homecage group for each animal. One case from the $0.3 \mathrm{~mA} \mid$ Remote group was discarded due to issues after multiple immunohistochemical essays, lowering the number of cases in this group down to 5. Counts from sections with tissue damage were excluded from analysis. 
Dos Santos Corrêa et al, 2021

\section{$4 \underline{\text { Statistical Analysis }}$}

\subsection{Behavior and Fos density for each ROI}

All descriptive and inferential statistical analysis for behavior and Fos density were done using JAMOVI (version 1.8) and Python. The conditioned fear response to context was quantified as the percent time the animal spent freezing during re-exposure to the training context. Behavioral results are expressed as the group mean percent freezing time \pm standard error of the mean (S.E.M). Immediate early gene activation results are expressed as the group mean normalized Fos density \pm S.E.M. All data were checked for the assumptions of normality and homogeneity of variances with the tests of Kolmogorov-Smirnov and Levene.

Behavioral data and Fos differences for each brain region were analyzed using ANOVAs, with the between subject factors being Footshock and Timepoint. The Tukey post hoc test was further used to identify significant differences when applicable. Significance for all tests described in this section was set at $p \leq 0.05$. Effect sizes for ANOVAs $\left(\eta^{2} \mathrm{p}\right)$ and post hoc tests (Cohen'd) are reported only when the test was found significant (" $\eta{ }^{2} p$ " values above 0.14 are considered large effects; values between 0.06 and 0.14 are considered moderate; and below 0.06, small) or in the Supplementary Material. Associations between freezing times and Fos density for each region were calculated using bicaudal Pearson correlations.

\subsection{Fos Coactivation analysis}

In experimental animal studies, neuronal activity is usually measured by evaluating changes in expression of immediate early genes such as $c$-fos or their products. Functional connectivity for these measures is assessed by calculating covariance across subjects, rather than within subjects (Careaga et al., 2019; Coelho et al., 2018; Worley et al., 2020). Within each of the six experimental groups of animals (retrieval test at recent or remote timepoints; Context Only, 0.3 and $1.0 \mathrm{~mA}$ ), all possible pairwise correlations between the Fos signal within the eight brain regions analyzed were determined by computing Spearman correlation coefficients (totaling 384 correlations). Each set of correlations was calculated from a vector of size 5-6, and were displayed as color-coded correlation matrices using Python. Fos coactivation (i.e., functional connectivity) was considered when the Spearman's rank correlation coefficient was below a threshold of one-tailed significance level of $\mathrm{p}<$ 0.05. To determine how Fos coactivation correlations differed by footshock intensity and timepoint, we contrasted mean $\mathrm{r}$ values between all ROIs for each group using a one-way Welch ANOVA. Games-Howell post hoc tests were used for multiple comparisons between groups. Significance for the ANOVA and post hoc tests described in this section was set at $p \leq 0.05$. To investigate effects of footshock intensity and timepoint on individual ROI by ROI correlations (i.e. is PrL more strongly correlated with BLA at the Remote timepoint versus Recent for each footshock intensity?), we 
109 contrasted the correlation of each condition to the others (all possible comparisons) using the Fisher $r$ 110 to $\mathrm{z}$ transformation and unicaudal $\mathrm{z}$ test to determine $p$ values for each contrast. The set of $\mathrm{p}$-values 111 were then globally adjusted to correct for multiple comparisons using Benjamini and Hochberg's false 112 discovery rate procedure (Worley et al., 2020) maintaining a false discovery rate of $25 \%$. Pairwise 113 ROI by ROI comparisons identified as significant using this method are summarized in Table 3. 


\section{Table 1}

Description of microscopy for each region of interest (ROI).

\begin{tabular}{|c|c|c|c|c|c|c|c|}
\hline Region & Microscope & Bregma Range & $\begin{array}{c}\text { Number of } \\
\text { bilateral frames } \\
\text { per ROI }\end{array}$ & $\begin{array}{l}\text { Size (pixels) } \\
\text { per photo }\end{array}$ & $\mu \mathrm{m}$ per pixel & Lens & $\begin{array}{c}\text { Median } \\
\text { bilateral } \\
\text { sections per } \\
\text { animal }\end{array}$ \\
\hline Hippocampus - Dentate Gyrus (DG) & Zeiss & {$[-2.28,-3.72]$} & 2 & 1388:1040 & 0,5 & $20 \mathrm{x}$ & 2 \\
\hline Hippocampus - CA3 & Zeiss & {$[-2.28,-3.72]$} & 1 & $1388: 1040$ & 0,5 & $20 \mathrm{x}$ & 2 \\
\hline Hippocampus - CA1 & Zeiss & {$[-2.28,-3.72]$} & 3 & $1388: 1040$ & 0,5 & $20 \mathrm{x}$ & 2 \\
\hline Prelimbic (PrL) & Leica & {$[4.2,2.52]$} & 1 & $3072: 2204$ & 0,3 & $10 \mathrm{x}$ & 3 \\
\hline Basolateral Amygdala (BLA) & Zeiss & {$[-1.56,-3.24]$} & 1 & $1388: 1040$ & 0,5 & $20 x$ & 2 \\
\hline Anterior Retrosplenial Cortex (aRSC) & Leica & {$[-2.28,-3.72]$} & 1 & 2410:1807 & 0,3 & $10 \mathrm{x}$ & 3 \\
\hline Anterior Cingulate Cortex (ACC) & Zeiss & {$[3.24,2.52]$} & 1 & $1388: 1040$ & 0,5 & $20 \mathrm{x}$ & 2 \\
\hline Anterior Insular Cortex (aIC) & Zeiss & {$[3.24,0.72]$} & 1 & 1388:1040 & 0,5 & $20 x$ & 3 \\
\hline
\end{tabular}

Note: The frames were taken from an interval of bregma coordinates that ranged from the most anterior to the most posterior for each brain region (column "Bregma"). Brain regions larger than the microscope's standard area were obtained in more than one frame (column "Number of bilateral frames per ROI"). Each animal had at least 1 and up to 3 bilateral brain sections to capture frames from; the column "Median bilateral sections per animal" shows the median number of bilateral sections per animal taken from all experimental groups in both recent and remote timepoints. 
Dos Santos Corrêa et al, 2021

\section{RESULTS}

\section{Remote memory retrieval is associated with higher neocortical functional connectivity}

The brains of a subset of rats used in a previous study (Dos Santos Corrêa et al., 2019) were used for analyses of the $c$-fos activity-dependent neuronal expression. Behavioral data of this subset of animals is representative of the main results previously published (Figure 1a). Increasing footshock intensity elicited higher freezing times in both recent [Footshock $\left(\mathrm{F}(2,14)=53.0, p<\square .001, \eta^{2} p=\right.$ $0.88)$ ] and remote timepoints [Footshock $\left.\left(\mathrm{F}(2,13)=24.60, p<\square .001, \eta^{2} p=0.79\right)\right]$. In both timepoints, rats from the C.O. groups showed significantly lower freezing when compared to the 0.3 (Recent, $p=0.01$; Remote, $p=0.02$ ) and $1.0 \mathrm{~mA}$ groups (Recent and Remote, $p<\square .001$ ). In addition, rats from the $0.3 \mathrm{~mA}$ group showed significantly lower freezing times when compared to the $1.0 \mathrm{~mA}$ group in both timepoints (Recent, $p<\square 0.001$; Remote, $p=0.003$, see table $\mathrm{S} 1$ for all post hoc results and effect sizes). These results were associated with remote contextual discrimination after low intensity training $(0.3 \mathrm{~mA})$ and remote contextual generalization after high intensity training $(1.0 \mathrm{~mA}$; DOS SANTOS CORRÊA et al., 2019). Post-training plasma CORT levels for each of the three footshock intensities in this subset of animals and inferential statistics can be seen in the supplementary material Fig S1 e Table S2.

Among the 8 brain regions analyzed, two-way ANOVAs revealed interaction effects for the aIC, PrL and aRSC. Figure 1 (b, c) shows quantification of Fos cells in the aIC and PrL, respectively. In the aIC we observed an increase of Fos signal only in the $1.0 \mathrm{~mA} /$ Remote group. The ANOVA showed significant effects for the Timepoint factor: $\mathrm{F}(1,27)=10.55, p<0.01, \eta^{2} p=0.28$ and Footshock * Timepoint interaction: $\mathrm{F}(2,27)=5.84, p<0.01, \eta^{2} p=0.30$, but not for the Footshock factor: $\mathrm{F}(2,27)=2.40, p=0.11$. Post hoc tests showed that the group of rats trained with $1.0 \mathrm{~mA}$ intensity and tested on the remote timepoint had a significant increase of immunoreactive cells when compared to rats trained with the same intensity and tested at the recent timepoint $(p<0.001, \mathrm{~d}=2.75$ $95 \% \mathrm{CI}=[1.34-4.16])$ and to rats only exposed to the context during training and tested at the recent $(p=0.003, \mathrm{~d}=2.5995 \% \mathrm{CI}=[1.15-4.03])$ or remote $(p=0.032, \mathrm{~d}=1.9895 \% \mathrm{CI}=[0.62-3.34])$ timepoints. All other comparisons were not significant $(p>0.09$, Fig. 2b). A similar increase was seen in the PrL. The ANOVA showed significant effects for the Footshock factor: $\mathrm{F}(2,26)=3.89, p=$ $0.033, \eta^{2} p=0.23$; Timepoint factor: $\mathrm{F}(1,26)=9.22, p<0.01, \eta^{2} p=0.26$ and Footshock $*$ Timepoint interaction: $\mathrm{F}(2,26)=6.58, p<0.01, \eta^{2} p=0.34$. Post hoc tests confirmed that the group of rats trained with $1.0 \mathrm{~mA}$ intensity and tested on the remote timepoint had a significant increase of immunoreactive cells when compared to all other groups $(p<0.042)$ whereas all other pairwise comparisons were not significant ( $p>0.70$, Fig. 2c). Table S3 and S4 report all post hoc comparisons for the aIC and PrL ANOVAs, with their effect sizes and 95\%CI. Even though the aRSC ANOVA showed an interaction effect $\left[\mathrm{F}(2,26)=3.77, p=0.04, \eta^{2} p=0.23\right.$, Table 2], post hoc tests did not 
Dos Santos Corrêa et al, 2021

confirm any differences between groups $(p>0.10$, Table S5). The Footshock and Timepoint factors for the aRSC ANOVA were also not significant $(p>0.29$, Table 2). Among the other 5 ROIs, twoway ANOVAs revealed main effects of Timepoint for the DG and CA1, but no main effect of Footshock or interactions (see Table 2 for full statistics). The ANOVAs also did not show any significant effects for the BLA, CA3 and ACC (Table 2).

To verify associations between freezing times and Fos activity for each ROI, we ran 16 bicaudal Pearson correlation tests for the recent and remote timepoints ( 8 per timepoint). The correlation tests did not reveal any significant correlation for the recent timepoint $(p>0.07$, see Fig. 1d) but revealed significant positive correlations for the BLA, PrL and aIC during remote retrieval ( $p$ $=0.024,0.034$ and 0.022, respectively, see Fig. 1d,e). All other correlation tests for the remote timepoint were not significant $(p>0.43)$.

In summary, regional immunoreactivity analyses showed an increased in Fos expression during the remote retrieval test in the aIC and PrL for the strong CFC. On the other hand, there was an overall increase in Fos cells for all groups during the remote CFC test in the DG and CA1 hippocampal subregions, regardless of footshock intensity. The correlation tests also suggest that Fos activity in the aIC, PrL and BLA during remote retrieval seems to be positively associated with higher freezing levels.

A

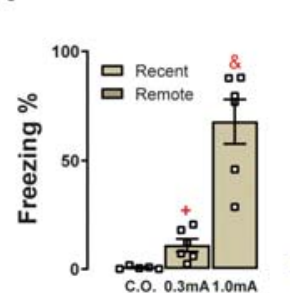

D

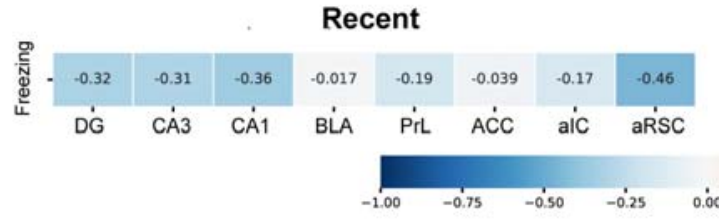

B

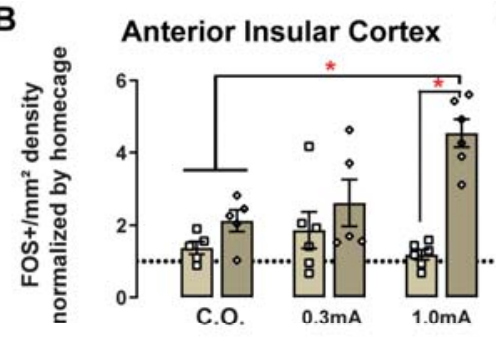

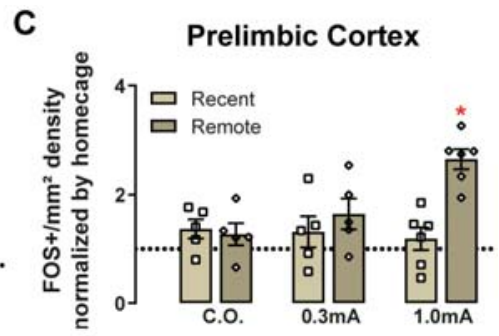

Remote
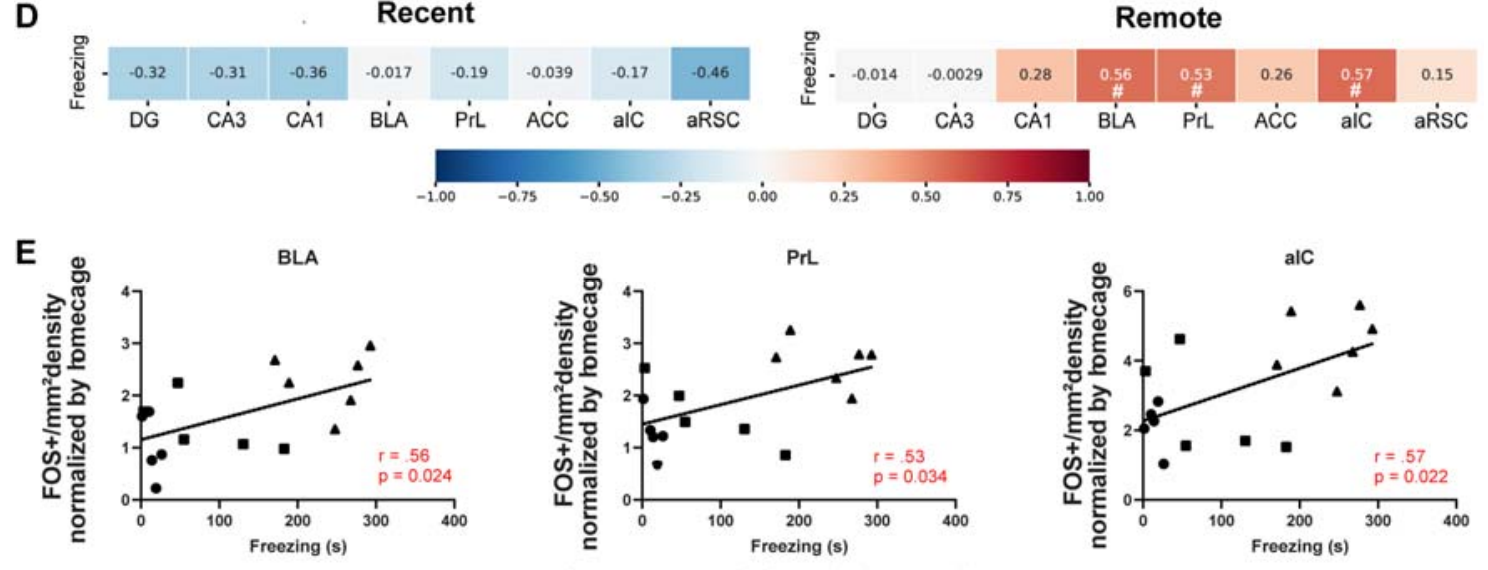

- Context only (C.O.) $=0.3 \mathrm{~mA} \cdot 1.0 \mathrm{~mA}$

52

Figure 1: Remote strong contextual fear conditioning retrieval is associated with increased Fos activity in the Anterior Insular and Pre limbic cortices. (a) Mean percentage of freezing time \pm Standard Error of the Mean (S.E.M) for rats submitted to the mild (0.3 mA footshocks) or strong (1.0 $\mathrm{mA}$ ) contextual fear conditioning (CFC) training and tested at the recent and remote timepoints (2 or 
28 days after training, respectively). Control animals were exposed to the context but did not receive any footshocks during training and were tested in the same timepoints (Context only, C.O.). Points represent raw data for each rat. $N=5$ for the $0.3 m A \mid$ Remote and C.O. groups, $N=6$ for all other groups. (+) $p<0.05$ when compared to the C.O. group in the same timepoint. (\&) $p<0.05$ when compared to the $0.3 \mathrm{~mA}$ and C.O. groups in the same timepoint. $(b, c)$ Mean Normalized Fos density \pm S.E.M. in the aIC and PrL, respectively. (*) $p<0.05$. Symbols show each animal's raw data. (d) Color-coded heatmap for Spearman correlations between freezing times and Fos activation in all ROIs during recent or remote retrieval. Red color represents strong, positive correlations, whereas blue color represents negative ones. (\#) significant bicaudal Pearson correlation $p<0.05$. (e) Correlations between freezing times during remote test sessions and Fos activation in the BLA, PrL and aIC; triangles correspond to the $1.0 \mathrm{~mA} \mid$ Remote group, squares to the $0.3 \mathrm{~mA} \mid$ Remote group and circles to the C.O.|Remote group.

\section{Pairwise co-variance between ROIs}

To map neuronal coactivity between brain areas implicated in either recent or remote memory retrieval, Fos expression was analyzed in the ROIs from each animal, and interregional Spearman correlations were calculated for each group (split by footshock intensity and timepoint, Figure 2a) (Careaga et al., 2019; Coelho et al., 2018; Worley et al., 2020). This analysis allows identifying pairs of brain regions in which normalized Fos density co-varies between subjects. Only those with high correlation were considered functionally connected (rho $\geq 0.81$ ). Results showed that Context Only groups show little to no coactivation between the ROIs in both timepoints. On the other hand, for the conditioned groups tested in the recent timepoint, many ROIs showed coactivation between themselves, in both footshock intensities. It is of note that the BLA and PrL show significant coactivation with all hippocampal subregions at this timepoint. Additionally, during remote retrieval, the $0.3 \mathrm{~mA}$ group shows sustained high coactivation between ROIs, similar to the pattern shown by the recent group, but with weakened coactivation between the BLA and PrL with hippocampal subregions. In contrast, the $1.0 \mathrm{~mA} \mid$ Remote group showed lessened coactivation between ROIs, having significant results only between the aIC and the DG-CA1.

To determine if coactivation between areas differed between experimental groups, we compared the averaged "r" values of all 8 ROIs using a Welch 1-way ANOVA (Worley et al., 2020). The ANOVA showed a significant effect of Group $\left[F(5,73.3)=23.2, p<0.001, \eta^{2} p=0.42\right.$ Fig. $\left.2 b\right]$. Averaged across all ROIs, we found more correlated activity in the $0.3 \mathrm{~mA} \mid$ Recent, $0.3 \mathrm{~mA} \mid$ Remote, $1.0 \mathrm{~mA} \mid$ Recent when compared to the C.O.|Recent, C.O.|Remote and 1.0mA|Remote groups (Fig. $2 \mathrm{~b}$, for all post hoc results and effect sizes see Table S6). To further investigate specific differences in coactivation between the shocked groups from the recent to the remote timepoint, data from each correlation was contrasted between timepoints using the Fisher $r$ to $z$ transformation and $\mathrm{z}$ test (Table 3 ). The test for the $0.3 \mathrm{~mA}$ groups indicated a significant increase in coactivation over time between the BLA-aRSC and from the DG-CA1. On the other hand, there was a significant decrease in connectivity in the 1.0mA groups between the DG-ACC, BLA-aRSC as well as between the aRSC- 
ACC and aRSC-BLA (Table 3). P-values of all $\mathrm{z}$ tests contrasts can be seen in Table S7. Z tests contrasting footshocks in the same timepoint were not done.

In summary, these results show that increasing footshock intensity in the CFC task elicited different trends in Fos coactivation between cortical and subcortical regions associated with contextual fear memories during remote retrieval, with a significant decrease of overall connectivity between ROIs in the $1.0 \mathrm{~mA} \mid$ Remote group compared to all other conditioned groups. Results from this group also show stronger decreases in coactivation between the aRSC and DG to the BLA and ACC when comparing the two timepoints. On the other hand, the $0.3 \mathrm{~mA}$ footshock intensity seems to maintain high connectivity between ROIs over time.

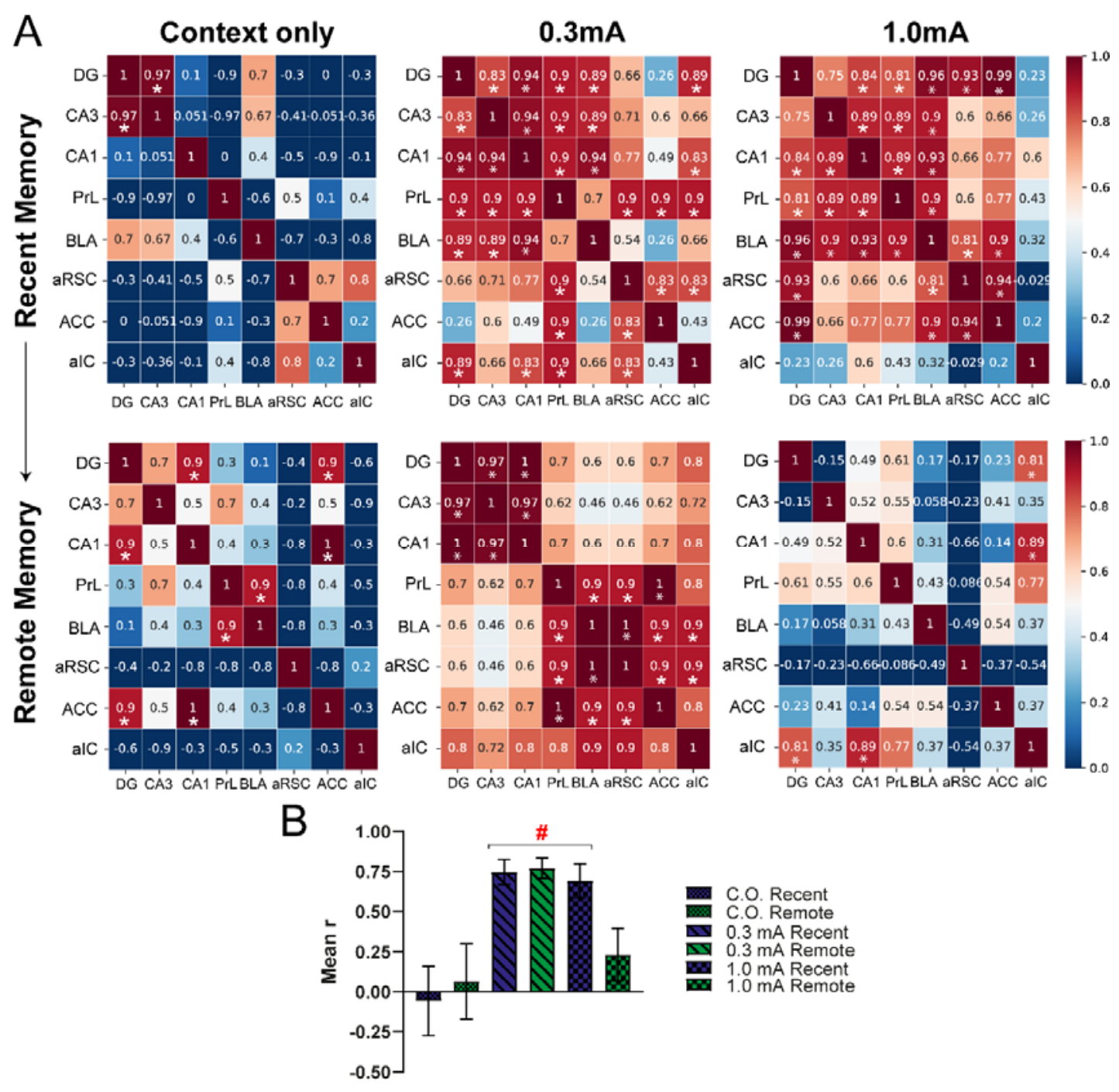


bioRxiv preprint doi: https://doi.org/10.1101/2021.12.22.473939- this version posted December 23,2021 . The copyright holder for this preprint (which was not certified by peer review) is the author/funder, who has granted bioRxiv a license to display the preprint in perpetuity. It is made available under aCC-BY-NC-ND 4.0 International license.

Dos Santos Corrêa et al, 2021

Figure 2: Increasing footshock intensity elicits different trends of functional connectivity between contextual memory brain regions during remote retrieval. (A) Correlation matrices showing pairwise co-variance between Regions of Interest (ROIs), or functional connectivity. Rats were submitted to the mild $(0.3 \mathrm{~mA}$ footshocks) or strong $(1.0 \mathrm{~mA})$ contextual fear conditioning $(C F C)$ training and tested at the recent and remote timepoints (2 or 28 days after training, respectively). Control animals were exposed to the context but did not receive any footshocks during training and were tested in the same timepoints (Context only, C.O.). Each correlation test compared vectors sized 5-6 (number of animals). Red color represents strong positive correlations, whereas blue color represents strong negative correlations $\left(^{*}\right) p<0.05$ in the unicaudal Spearman correlation test. $(B)$ Mean $r$ values $\pm 95 \%$ confidence interval across all ROIs per group. (\#) $p<0.001$ in comparison to the 1.0mA|Remote and Context Only groups (recent and remote timepoints). 
Table 2

Descriptive (mean normalized Fos density \pm S.E.M) and inferential statistics (ANOVAs) for other analyzed brain regions.

\begin{tabular}{|c|c|c|c|c|c|c|c|c|c|c|c|}
\hline \multicolumn{2}{|c|}{ Brain Region } & \multicolumn{2}{|c|}{ Context-only } & \multicolumn{2}{|l|}{$0.3 \mathrm{~mA}$} & \multicolumn{2}{|l|}{$1.0 \mathrm{~mA}$} & \multicolumn{4}{|l|}{ ANOVA } \\
\hline & & Recent & Remote & Recent & Remote & Recent & Remote & Factor & $\mathrm{F}$ & $\mathrm{df}$ & $p$-value \\
\hline DG & Mean & 1.50 & 1.94 & 1.31 & 1.74 & 0.877 & 2.22 & Footshock & 0.119 & 2,27 & 0.888 \\
\hline & S.E.M \pm & 0.415 & 0.549 & 0.515 & 0.528 & 0.204 & 0.280 & Timepoint & 4.485 & 1,27 & 0.044* \\
\hline \multirow[t]{3}{*}{$\mathrm{CA3}$} & Mean & 1.44 & 1.29 & 1.12 & 0.948 & 0.922 & 1.48 & Footshock & 0.326 & 2,27 & 0.725 \\
\hline & S.E.M \pm & 0.459 & 0.553 & 0.388 & 0.509 & 0.310 & 0.172 & Timepoint & 0.062 & 1,27 & 0.805 \\
\hline & $\mathrm{N}$ & 5 & 5 & 6 & 5 & 6 & 6 & Footshock*Timepoint & 0.552 & 2,27 & 0.582 \\
\hline & S.E.M \pm & 0.528 & 0.886 & 0.508 & 0.904 & 0.217 & 0.915 & Timepoint & 4.517 & 1,27 & 0.043* \\
\hline & $\mathrm{N}$ & 5 & 5 & 6 & 5 & 6 & 6 & Footshock*Timepoint & 1.898 & 2,27 & 0.169 \\
\hline \multirow[t]{3}{*}{ BLA } & Mean & 1.11 & 1.03 & 1.13 & 1.43 & 1.11 & 2.29 & Footshock & 2.27 & 2,27 & 0.123 \\
\hline & S.E.M \pm & 0.407 & 0.276 & 0.153 & 0.238 & 0.424 & 0.238 & Timepoint & 3.48 & 1,27 & 0.073 \\
\hline & $\mathrm{N}$ & 5 & 5 & 6 & 5 & 6 & 6 & Footshock*Timepoint & 2.30 & 2,27 & 0.120 \\
\hline aRSC & Mean & 1.52 & 0.971 & 1.38 & 1.65 & 1.00 & 2.03 & Footshock & 0.557 & 2,26 & 0.580 \\
\hline & S.E.M \pm & 0.155 & 0.233 & 0.200 & 0.275 & 0.243 & 0.370 & Timepoint & 2.12 & 1,27 & 0.157 \\
\hline & $\mathrm{N}$ & 5 & 5 & 6 & 5 & 6 & 6 & Footshock*Timepoint & 1.76 & 2,27 & 0.192 \\
\hline
\end{tabular}




\section{Table 3}

Summary of significant pairwise ROI by ROI comparisons.

\begin{tabular}{lllr}
\hline Footshock & ROI - ROI & Contrast & $p$-value \\
\hline $0.3 \mathrm{~mA}$ & BLA-aRSC & REC $<$ REM & $<0.001$ \\
& DG-CA1 & REC $<$ REM & 0.01 \\
\hline $1.0 \mathrm{~mA}$ & DG-ACC & REC $>$ REM & $<0.01$ \\
& DG-BLA & REC $>$ REM & 0.02 \\
& DG-aRSC & REC $>$ REM & 0.01 \\
& BLA-aRSC & REC $>$ REM & 0.02 \\
& ACC-aRSC & REC $>$ REM & $<0.01$ \\
\hline
\end{tabular}

Note: $\mathrm{REC}=$ recent timepoint; REM = remote timepoint. The timepoint that had higher correlation between ROIs is shown in bold.

\section{DISCUSSION}

Our laboratory recently showed that stronger CFC trainings ( $3 \mathrm{x} 0.6$ or $1.0 \mathrm{~mA}$ footshocks) elicited time-dependent fear generalization, whereas mild CFC trainings ( $3 \mathrm{x} 0.3 \mathrm{~mA}$ footshocks) prompted remote contextual discrimination (Dos Santos Corrêa et al., 2019). We suggested that these results were due to different systems consolidation processes that occur after a mild or strong CFC, which is now further confirmed here. We also observed that higher CORT levels were associated with time-dependent fear generalization. Now, we demonstrate that strong CFC training elicits higher Fos activation of the aIC and PrL during remote retrieval, and Fos activity in these regions and in the BLA at this timepoint is associated with freezing response to the training context. Rats trained with the strong CFC protocol are also the ones with higher levels of post-training corticosterone (Fig. S2). Also, remote retrieval after mild CFC training seems to induce sustained high functional connectivity, whereas strong CFC training elicits lower functional connectivity between the ROIs at this timepoint. Lastly, strong CFC remote retrieval seems to have diminished connectivity between the aRSC and DG to the BLA and ACC compared to the same training intensity in recent retrieval, suggesting a possible decrease in engagement of the retrosplenial/hippocampus network overtime after stronger trainings. The overall data suggest that increasing training intensity during memory acquisition results in differential processes of systems consolidation that can be further associated with either fear discrimination after mild CFC or generalized fear after strong CFC (Dos Santos Corrêa et al., 2019). Fos levels reported here are similar to those conveyed in past studies that have analyzed neuronal activity after recent and remote contextual fear memory retrieval (Silva et al., 2019; Tayler et al., 2013).

Wheeler and colleagues (2013) showed that there is increased co-variance between neocortical and hippocampal regions after remote retrieval, in contrast to what is found after recent 
23 retrieval when co-variance seems to be restricted to a hippocampal-centered network (Wheeler et al.,

24 2013). Earlier lesion studies indicated that remote retrieval becomes independent of the hippocampus,

25 and the memory trace seems to lose some of the contextual details that are necessary for

26 discriminative retrieval in this process (Frankland and Bontempi, 2005). Nonetheless, this hypothesis

27 has been challenged by several studies showing that the hippocampus remains engaged during remote

28 memory retrieval (Albo and Gräff, 2018; Sutherland and Lehmann, 2011). The connectivity studies

29 mentioned above strengthen this hypothesis and our results agree with them. Our data suggest that

30 some neocortical regions become more engaged during memory retrieval at remote timepoints (but

31 only for strong CFC) and the hippocampus may maintain its functional connectivity to other ROIs

32 (mild CFC) at this same timepoint. It is possible that, in the earlier lesion studies, the strengthening of

33 neocortical functional connectivity over time compensated for the absence of a functional

34 hippocampus during remote retrieval, and may be the reason why temporarily inactivating the

35 hippocampus before remote retrieval tests did not impair freezing levels (Quillfeldt, 2019).

36 Our results suggest an association between freezing behavior and Fos activity in the PrL, BLA

37 and aIC during remote CFC retrieval, possibly indicating that engram cells allocated in these regions

38 during encoding may mature over time into a "contextual fear network". Interestingly, these regions

39 are associated with the SN (with some divergence over which region specifically, the ACC or PrL, is

40 indeed part of the network in rodents) (Hermans et al., 2014a; Seeley, 2019). The dHPC, aRSC and

41 ACC in rodents are associated with the DMN-like network, an analogous of the DMN in humans,

42 which is inversely correlated with SN activation (Zerbi et al., 2015). Therefore, we hypothesize that

43 strong contextual fear memory engrams are allocated during the encoding phase in SN associated

44 regions, especially in the aIC and PrL, via increased noradrenergic and glucocorticoid activity in these

45 regions (Schwabe, 2017).

46 Neuroimaging studies in humans have associated increased coherence between the aIC,

$47 \mathrm{mPFC}$ and amygdala in response to stressful events or treatment with noradrenergic or glucocorticoid

48 agonists (Quaedflieg and Schwabe, 2018). Correspondingly, recent studies with PTSD patients have

49 indicated that they show an exacerbated processing of interoceptive/arousal stimuli in the aIC

50 (Nicholson et al., 2020), increased connectivity between the ACC and the aIC (Caseras et al., 2013)

51 and also that these brain regions are associated with generalized threat detection in these patients

52 (Berg et al., 2020). Webler and colleagues (2021) described that an increasing gradient of

53 generalization is associated with higher connectivity between the aIC, dorsomedial PFC (the

54 analogous of the rodent PrL), dorsal ACC along with other regions, and also with a decrease in

55 connectivity in nodes of the DMN as generalization increased (Webler et al., 2021). Therefore, it is

56 possible that strongly arousing events (i.e. strong CFC training) produce an enduring memory engram

57 in neocortical regions associated with the SN, which are initially silent and mature over time (Josselyn 
58 and Tonegawa, 2020), undergoing systems consolidation. On the other hand, a different process 59 seems to occur after mildly arousing events, maintaining memory dependence on DMN-like regions.

60 Recent studies in rodents showed that noradrenergic activation of the BLA during memory 61 consolidation influences neural plasticity in the aIC and PrL and enhances recognition memory 62 (Barsegyan et al., 2019). Likewise, activation of glucocorticoid receptors in the aIC is associated with 63 enhanced contextual fear memory consolidation (Fornari et al., 2012). These results suggest that 64 aversive events may recruit these regions during memory encoding. However, is this recruitment 65 arousal-dependent? It seems likely to be. The engagement of the aIC in the retrieval of CFC is 66 intensity dependent, as its post-training functional inhibition impairs recent retrieval after stronger 67 trainings (Alves et al., 2013) but not weaker ones (de Paiva et al., 2021). This dependence on the 68 training intensity was also reported in the PrL in mice (Matos et al., 2019). These results together 69 agree with ours and suggest that the aIC and PrL may be recruited differentially for the consolidation 70 of contextual memories after mild or stronger aversive events. Lastly, the increase in functional 71 connectivity between DG and CA1 in the $1.0 \mathrm{~mA} \mid$ Remote group to the aIC is intriguing, considering 72 that there are few input and output projections to and from the aIC and the hippocampus (Gehrlach et 73 al., 2020). Possibly, functional connectivity between these two regions may be explained by their 74 mutual projections to the medial prefrontal cortex, suggesting that a mPFC-aIC pathway is engaged 75 during strong CFC remote retrieval. Barsegyan and colleagues (2019) provide evidence that the PrL 76 may serve as a site of integration between the hippocampal and insular systems (Barsegyan et al., 77 2019) and this circuit might also be relevant for generalized remote CFC retrieval.

A limitation of our study is that we have only quantified Fos from animals that were tested in the training context and not in a novel one. More experiments are needed to investigate neuronal activation in the ROIs that are engaged during exposure to novel contexts after mild or strong CFC. It

81 is possible that the fear network investigated here is engaged only when the animal is exposed to the 82 training context, therefore all associations between generalized fear and loss of functional 83 connectivity between the ROIs studied here are second-order inferences. Moreover, the lack of 84 differences between rats trained with or without footshocks for most ROIs is noteworthy. Even though 85 we expected to see differences in activation in the hippocampal and amygdalar regions, this difference 86 was not confirmed by the data. Another study that also quantified Fos expression has found a lack of 87 difference between these groups in the dorsal hippocampus at the remote timepoint (Silva et al., 2019) 88 but this study and others have found significant differences in neocortical and amygdalar regions that 89 we did not, especially in the ACC and aRSC (Aceti et al., 2015; Silva et al., 2019). These studies, 90 however, used several different parameters that could account for the divergent results, such as the use 91 of a different species (mice), stronger training parameters (5 or 30 footshocks) or a different behavioral task (inhibitory avoidance). 
93

To conclude, we found correlational evidence between an increase of Fos activation in regions associated with the $\mathrm{SN}$ after strong $\mathrm{CFC}$ remote retrieval. Hence, we infer that increasing arousal levels (with contiguous increased glucocorticoid signaling in the brain) during memory encoding elicit different processes of systems consolidation, resulting in the recruitment of different clusters of brain regions in response to contextual fear conditioning. Strong CFC elicits the release of higher levels of CORT (Dos Santos Correa et al., 2019), and maybe noradrenaline, possibly recruiting regions associated with the SN (PrL and aIC). On the other hand, mild CFC training would not reach the necessary levels of arousal that allow engram allocation in these areas, preserving contextuallyspecific remote retrieval (Dos Santos Corrêa et al., 2021; Pedraza et al., 2016) and the forementioned dependence on the DMN-like (Tonegawa et al., 2018). The highest footshock intensity training was previously associated with time-dependent fear generalization, implicating a possible relationship between increased neuronal activity in the SN to generalized freezing (Dos Santos Corrêa et al., 2019). Overgeneralization of fear is one of the most debilitating symptoms of PTSD and generalized anxiety disorder, consequently our results indicate the necessity of targeting the SN in future neuromodulatory treatments for these disorders.

\section{Acknowledgements}

We thank the valuable support from Bruna de Oliveira, Thaisy Moraes Costa and Gabriel Leir Gandra for helping in some experiments. We also thank the work done by the technicians that manage the vivarium. The authors would like to thank the members of the Research group in Neurobiology of Learning and Memory at UFABC (MANAs) for scientific advice and discussion.

\section{Declaration of Competing Interests}

The authors report no conflicts of interest in this work.

\section{Research data for this article}

The data used for all analyses in this study can be found at https://data.mendeley.com/datasets/kztbft87y6.

\section{Funding}

This work was financially supported by the São Paulo Research Foundation (FAPESP) grant \#2017/03820-0 (RVF), FAPESP fellowship \#2017/24012-9 (MdSC), FAPESP grant \#2011/10062-8 
(PAT), and Conselho Nacional de Desenvolvimento Científico e Tecnológico (CNPQ) grant \#429894/2016-3 (TLF). Students GDVG and TSAT were partially funded by fellowships from Universidade Federal do ABC (UFABC).

\section{Author Statement}

MdSC helped conceptualizing, doing experiments, curating data, doing formal analysis and experiments, planning methodology and writing the original draft. GDVG, IAFF, and TSAT helped doing experiments and reviewing the manuscript. PAT, TLF and RVF helped conceptualizing, acquiring funds, planning methodology, reviewing the manuscript, and supervising the project. 
Dos Santos Corrêa et al, 2021

\section{REFERENCES}

[dataset] dos Santos Corrêa, M., Barbara dos Santos, V., Gabriel David Vieira, G., Joselisa Péres Queiroz, de P., Paula Ayako, T., Fornari, R.V., 2019. Relationship between footshock intensity, post-training corticosterone release and contextual fear memory specificity over time (raw data). Mendeley Data, v5. https://doi.org/10.17632/n5zdf72x6s.5

Aceti, M., Vetere, G., Novembre, G., Restivo, L., Ammassari-Teule, M., 2015. Progression of activity and structural changes in the anterior cingulate cortex during remote memory formation. Neurobiol. Learn. Mem. 123, 67-71. https://doi.org/10.1016/j.nlm.2015.05.003

Albo, Z., Gräff, J., 2018. The mysteries of remote memory. Philos. Trans. R. Soc. Lond. B. Biol. Sci. 373, 20170029. https://doi.org/10.1098/rstb.2017.0029

Alves, F.H.F., Gomes, F. V., Reis, D.G., Crestani, C.C., Corrêa, F.M.A., Guimarães, F.S., Resstel, L.B.M., 2013. Involvement of the insular cortex in the consolidation and expression of contextual fear conditioning. Eur. J. Neurosci. 38, 2300-7. https://doi.org/10.1111/ejn.12210

Arnsten, A.F.T., 2009. Stress signalling pathways that impair prefrontal cortex structure and function. Nat. Rev. Neurosci. 10, 410-422. https://doi.org/10.1038/nrn2648

Atucha, E., Vukojevic, V., Fornari, R. V., Ronzoni, G., Demougin, P., Peter, F., Atsak, P., Coolen, M.W., Papassotiropoulos, A., McGaugh, J.L., de Quervain, D.J.-F., Roozendaal, B., 2017. Noradrenergic activation of the basolateral amygdala maintains hippocampus-dependent accuracy of remote memory. Proc. Natl. Acad. Sci. 201710819. https://doi.org/10.1073/pnas.1710819114

Barry, D.N., Maguire, E.A., 2019. Remote Memory and the Hippocampus: A Constructive Critique. Trends Cogn. Sci. 23, 128-142. https://doi.org/10.1016/j.tics.2018.11.005

Barsegyan, A., Mirone, G., Ronzoni, G., Guo, C., Song, Q., van Kuppeveld, D., Schut, E.H.S., Atsak, P., Teurlings, S., McGaugh, J.L., Schubert, D., Roozendaal, B., 2019. Glucocorticoid enhancement of recognition memory via basolateral amygdala-driven facilitation of prelimbic cortex interactions. Proc. Natl. Acad. Sci. U. S. A. 116, 7077-7082.

https://doi.org/10.1073/pnas.1901513116

Berg, H., Ma, Y., Rueter, A., Kaczkurkin, A., Burton, P.C., Deyoung, C.G., Macdonald, A.W., Sponheim, S.R., Lissek, S.M., 2020. Salience and central executive networks track overgeneralization of conditioned-fear in post-traumatic stress disorder. Psychol. Med. https://doi.org/10.1017/S0033291720001166

Careaga, M.B.L., Girardi, C.E.N., Suchecki, D., 2019. Variability in response to severe stress: highly reactive rats exhibit changes in fear and anxiety-like behavior related to distinct neuronal coactivation patterns. Behav. Brain Res. 373, 112078. https://doi.org/10.1016/j.bbr.2019.112078

Caseras, X., Murphy, K., Mataix-Cols, D., López-Solà, M., Soriano-Mas, C., Ortriz, H., Pujol, J., Torrubia, R., 2013. Anatomical and functional overlap within the insula and anterior cingulate cortex during interoception and phobic symptom provocation. Hum. Brain Mapp. 34, 1220-9. https://doi.org/10.1002/hbm.21503

Coelho, C.A.O., Ferreira, T.L., Kramer-Soares, J.C., Sato, J.R., Oliveira, M.G.M., 2018. Network supporting contextual fear learning after dorsal hippocampal damage has increased dependence on retrosplenial cortex. PLoS Comput. Biol. 14, e1006207. https://doi.org/10.1371/journal.pcbi.1006207

de Paiva, J.P.Q., Bueno, A.P.A., Dos Santos Corrêa, M., Oliveira, M.G.M., Ferreira, T.., Fornari, 
R.V., 2021. The posterior insular cortex is necessary for the consolidation of tone fear conditioning. Neurobiol. Learn. Mem. 179, 107402. https://doi.org/10.1016/j.nlm.2021.107402

DeNardo, L.A., Liu, C.D., Allen, W.E., Adams, E.L., Friedmann, D., Fu, L., Guenthner, C.J., TessierLavigne, M., Luo, L., 2019. Temporal evolution of cortical ensembles promoting remote memory retrieval. Nat. Neurosci. 22, 460-469. https://doi.org/10.1038/s41593-018-0318-7

Dos Santos Corrêa, M., Vaz, B. dos S., Grisanti, G.D.V., de Paiva, J.P.Q., Tiba, P.A., Fornari, R.V., 2019. Relationship between footshock intensity, post-training corticosterone release and contextual fear memory specificity over time. Psychoneuroendocrinology 110, 104447. https://doi.org/10.1016/j.psyneuen.2019.104447

Dos Santos Corrêa, M., Vaz, B. dos S., Menezes, B.S., Ferreira, T.L., Tiba, P.A., Fornari, R.V., 2021. Corticosterone differentially modulates time-dependent fear generalization following mild or moderate fear conditioning training in rats. Neurobiol. Learn. Mem. 184, 107487. https://doi.org/10.1016/j.nlm.2021.107487

Fornari, R. V., Wichmann, R., Atucha, E., Desprez, T., Eggens-Meijer, E., Roozendaal, B., 2012. Involvement of the insular cortex in regulating glucocorticoid effects on memory consolidation of inhibitory avoidance training. Front. Behav. Neurosci. 6, 10. https://doi.org/10.3389/fnbeh.2012.00010

Frankland, P.W., Bontempi, B., 2005. The organization of recent and remote memories. Nat. Rev. Neurosci. 6, 119-30. https://doi.org/10.1038/nrn1607

Frankland, P.W., Bontempi, B., Talton, L.E., Kaczmarek, L., Silva, A.J., 2004. The Involvement of the Anterior Cingulate Cortex in Remote Contextual Fear Memory. Science (80-. ). 304, 881883. https://doi.org/10.1126/science.1094804

Gazarini, L., Stern, C.A., Takahashi, R.N., Bertoglio, L.J., 2021. Interactions of Noradrenergic, Glucocorticoid and Endocannabinoid Systems Intensify and Generalize Fear Memory Traces. Neuroscience. https://doi.org/10.1016/j.neuroscience.2021.09.012

Gehrlach, D.A., Weiand, C., Gaitanos, T.N., Cho, E., Klein, A.S., Hennrich, A.A., Conzelmann, K., Gogolla, N., 2020. A whole-brain connectivity map of mouse insular cortex. Elife 9, 2020.02.10.941518. https://doi.org/10.7554/eLife.55585

Goshen, I., Brodsky, M., Prakash, R., Wallace, J., Gradinaru, V., Ramakrishnan, C., Deisseroth, K., 2011. Dynamics of Retrieval Strategies for Remote Memories. Cell 147, 678-689. https://doi.org/10.1016/j.cell.2011.09.033

Hermans, E.J., Battaglia, F.P., Atsak, P., De Voogd, L.D., Fernández, G., Roozendaal, B., 2014a. How the amygdala affects emotional memory by altering brain network properties. Neurobiol. Learn. Mem. 112, 2-16. https://doi.org/10.1016/j.nlm.2014.02.005

Hermans, E.J., Henckens, M.J.A.G.A.G., Joëls, M., Fernández, G., 2014b. Dynamic adaptation of large-scale brain networks in response to acute stressors. Trends Neurosci. 37, 304-314. https://doi.org/10.1016/j.tins.2014.03.006

Ji, M., Xia, J., Tang, X., Yang, J., 2018. Altered functional connectivity within the default mode network in two animal models with opposing episodic memories. PLoS One 13, e0202661. https://doi.org/10.1371/journal.pone.0202661

Josselyn, S.A., Tonegawa, S., 2020. Memory engrams: Recalling the past and imagining the future. Science 367. https://doi.org/10.1126/science.aaw4325 
Mahan, A.L., Ressler, K.J., 2012. Fear conditioning, synaptic plasticity and the amygdala: Implications for posttraumatic stress disorder. Trends Neurosci. 35, 24-35. https://doi.org/10.1016/j.tins.2011.06.007

Mandino, F., Vrooman, R.M., Foo, H.E., Yeow, L.Y., Bolton, T.A.W., Salvan, P., Teoh, C.L., Lee, C.Y., Beauchamp, A., Luo, S., Bi, R., Zhang, J., Lim, G.H.T., Low, N., Sallet, J., Gigg, J., Lerch, J.P., Mars, R.B., Olivo, M., Fu, Y., Grandjean, J., 2021. A triple-network organization for the mouse brain. Mol. Psychiatry 1-8. https://doi.org/10.1038/s41380-021-01298-5

Matos, M.R., Visser, E., Kramvis, I., van der Loo, R.J., Gebuis, T., Zalm, R., Rao-Ruiz, P., Mansvelder, H.D., Smit, A.B., van den Oever, M.C., 2019. Memory strength gates the involvement of a CREB-dependent cortical fear engram in remote memory. Nat. Commun. 10, 2315. https://doi.org/10.1038/s41467-019-10266-1

Moscarello, J.M., Maren, S., 2018. Flexibility in the face of fear: hippocampal-prefrontal regulation of fear and avoidance. Curr. Opin. Behav. Sci. 19, 44-49. https://doi.org/10.1016/j.cobeha.2017.09.010

Moscovitch, M., Cabeza, R., Winocur, G., Nadel, L., 2016. Episodic memory and beyond: The hippocampus and neocortex in transformation. Annu. Rev. Psychol. 67, 105-134. https://doi.org/10.1146/annurev-psych-113011-143733

Nicholson, A.A., Harricharan, S., Densmore, M., Neufeld, R.W.J., Ros, T., McKinnon, M.C., Frewen, P.A., Théberge, J., Jetly, R., Pedlar, D., Lanius, R.A., 2020. Classifying heterogeneous presentations of PTSD via the default mode, central executive, and salience networks with machine learning. NeuroImage Clin. 27, 102262. https://doi.org/10.1016/j.nicl.2020.102262

Paxinos, G., Watson, C.R., 2007. The Rat Brain in Stereotaxic Coordinates, 6th Editio. ed. Academic Press.

Pedraza, L.K., Sierra, R.O., Boos, F.Z., Haubrich, J., Quillfeldt, J.A., de Oliveira Alvares, L., 2016. The dynamic nature of systems consolidation: Stress during learning as a switch guiding the rate of the hippocampal dependency and memory quality. Hippocampus 26, 362-371. https://doi.org/10.1002/hipo.22527

Quaedflieg, C.W.E.M., Schwabe, L., 2018. Memory dynamics under stress. Memory 26, 364-376. https://doi.org/10.1080/09658211.2017.1338299

Quillfeldt, J.A., 2019. Temporal Flexibility of Systems Consolidation and the Synaptic Occupancy/Reset Theory (SORT): Cues About the Nature of the Engram. Front. Synaptic Neurosci. 11, 1. https://doi.org/10.3389/fnsyn.2019.00001

Redondo, R.L., Kim, J., Arons, A.L., Ramirez, S., Liu, X., Tonegawa, S., 2014. Bidirectional switch of the valence associated with a hippocampal contextual memory engram. Nature 513, 426-430. https://doi.org/10.1038/nature13725

Roozendaal, B., Mirone, G., 2020. Opposite Effects of Noradrenergic and Glucocorticoid Activation on Accuracy of Episodic-like Memory. Psychoneuroendocrinology 104588. https://doi.org/10.1016/j.psyneuen.2020.104588

Roy, D.S., Arons, A., Mitchell, T.I., Pignatelli, M., Ryan, T.J., Tonegawa, S., 2016. Memory retrieval by activating engram cells in mouse models of early Alzheimer's disease. Nature 531, 508-512. https://doi.org/10.1038/nature17172

Sano, Y., Shobe, J.L., Zhou, M., Huang, S., Shuman, T., Cai, D.J., Golshani, P., Kamata, M., Silva, A.J., 2014. CREB regulates memory allocation in the insular cortex. Curr. Biol. 24, 2833-2837. 
Dos Santos Corrêa et al, 2021

https://doi.org/10.1016/j.cub.2014.10.018

Schwabe, L., 2017. Memory under stress: from single systems to network changes. Eur. J. Neurosci. 45, 478-489. https://doi.org/10.1111/ejn.13478

Seeley, W.W., 2019. The salience network: A neural system for perceiving and responding to homeostatic demands. J. Neurosci. 39, 9878-9882. https://doi.org/10.1523/JNEUROSCI.113817.2019

Silva, B.A., Burns, A.M., Gräff, J., 2019. A cFos activation map of remote fear memory attenuation. Psychopharmacology (Berl). 236, 369-381. https://doi.org/10.1007/s00213-018-5000-y

Sutherland, R.J., Lehmann, H., 2011. Alternative conceptions of memory consolidation and the role of the hippocampus at the systems level in rodents. Curr. Opin. Neurobiol. 21, 446-451. https://doi.org/10.1016/j.conb.2011.04.007

Tanaka, K.Z., Pevzner, A., Hamidi, A.B., Nakazawa, Y., Graham, J., Wiltgen, B.J., 2014. Cortical Representations Are Reinstated by the Hippocampus during Memory Retrieval. Neuron 84, 347-354. https://doi.org/10.1016/j.neuron.2014.09.037

Tayler, K.K., Tanaka, K.Z., Reijmers, L.G., Wiltgen, B.J., 2013. Reactivation of neural ensembles during the retrieval of recent and remote memory. Curr. Biol. 23, 99-106. https://doi.org/10.1016/j.cub.2012.11.019

Tonegawa, S., Morrissey, M.D., Kitamura, T., 2018. The role of engram cells in the systems consolidation of memory. Nat. Rev. Neurosci. 19, 485-498. https://doi.org/10.1038/s41583-0180031-2

Upadhyay, J., Baker, S.J., Chandran, P., Miller, L., Lee, Y., Marek, G.J., Sakoglu, U., Chin, C.L., Luo, F., Fox, G.B., Day, M., 2011. Default-Mode-Like Network Activation in Awake Rodents. PLoS One 6. https://doi.org/10.1371/journal.pone.0027839

Wang, S.-H., Teixeira, C.M., Wheeler, A.L., Frankland, P.W., 2009. The precision of remote context memories does not require the hippocampus. Nat. Neurosci. 12, 253-255. https://doi.org/10.1038/nn.2263

Webler, R.D., Berg, H., Fhong, K., Tuominen, L., Holt, D.J., Morey, R.A., Lange, I., Burton, P.C., Fullana, M.A., Radua, J., Lissek, S., 2021. The neurobiology of human fear generalization: meta-analysis and working neural model. Neurosci. Biobehav. Rev. 128, 421-436. https://doi.org/10.1016/j.neubiorev.2021.06.035

Wheeler, A.L., Teixeira, C.M., Wang, A.H., Xiong, X., Kovacevic, N., Lerch, J.P., McIntosh, A.R., Parkinson, J., Frankland, P.W., 2013. Identification of a Functional Connectome for Long-Term Fear Memory in Mice. PLoS Comput. Biol. 9. https://doi.org/10.1371/journal.pcbi.1002853

Winocur, G., Moscovitch, M., Bontempi, B., 2010. Memory formation and long-term retention in humans and animals: Convergence towards a transformation account of hippocampalneocortical interactions. Neuropsychologia 48, 2339-2356. https://doi.org/10.1016/j.neuropsychologia.2010.04.016

Worley, N.B., Everett, S.R., Foilb, A.R., Christianson, J.P., 2020. Functional networks activated by controllable and uncontrollable stress in male and female rats. Neurobiol. Stress 13, 100233. https://doi.org/10.1016/j.ynstr.2020.100233

Zerbi, V., Floriou-Servou, A., Markicevic, M., Vermeiren, Y., Sturman, O., Privitera, M., von Ziegler, L., Ferrari, K.D., Weber, B., De Deyn, P.P., Wenderoth, N., Bohacek, J., 2019. Rapid 
Reconfiguration of the Functional Connectome after Chemogenetic Locus Coeruleus Activation. Neuron 103, 702-718.e5. https://doi.org/10.1016/j.neuron.2019.05.034

Zerbi, V., Grandjean, J., Rudin, M., Wenderoth, N., 2015. Mapping the mouse brain with rs-fMRI: An optimized pipeline for functional network identification. Neuroimage 123, 11-21. https://doi.org/10.1016/j.neuroimage.2015.07.090

Zhang, Y., Fukushima, H., Kida, S., 2011. Induction and requirement of gene expression in the anterior cingulate cortex and medial prefrontal cortex for the consolidation of inhibitory avoidance memory. Mol. Brain 4, 4. https://doi.org/10.1186/1756-6606-4-4 
bioRxiv preprint doi: https://doi.org/10.1101/2021.12.22.473939; this version posted December 23, 2021. The copyright holder for this preprint

(which was not certified by peer review) is the author/funder, who has granted bioRxiv a license to display the preprint in perpetuity. It is made available under aCC-BY-NC-ND 4.0 International license.

Context only \begin{tabular}{l|l|l|l|l|l|l|l} 
DG - 1 & 0.97 & 0.1 & -0.9 & 0.7 & -0.3 & 0 & -0.3 \\
\hline
\end{tabular}

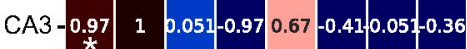
\begin{tabular}{llllllll|l|l} 
CA1 -0.1 & 0.051 & 1 & 0 & 0.4 & -0.5 & -0.9 & -0.1
\end{tabular} \begin{tabular}{ll|l|l|l|l|l|l} 
PrL -0.9 & -0.97 & 0 & 1 & -0.6 & 0.5 & 0.1 & 0.4
\end{tabular} \begin{tabular}{lllllll|l|l|l} 
BLA -0.7 & 0.67 & 0.4 & -0.6 & 1 & -0.7 & -0.3 & -0.8 \\
\hline
\end{tabular} \begin{tabular}{ll|l|l|l|l|l|l|} 
aRSC -0.3 & -0.41 & -0.5 & 0.5 & -0.7 & 1 & 0.7 & 0.8 \\
\hline
\end{tabular} \begin{tabular}{ll|l|l|l|l|l|l} 
ACC - 0 & 0.051 & -0.9 & 0.1 & -0.3 & 0.7 & 1 & 0.2
\end{tabular} \begin{tabular}{l|l|l|l|l|l|l|l} 
alC -0.0 .3 & -0.36 & -0.1 & 0.4 & -0.8 & 0.8 & 0.2 & 1
\end{tabular} DG CA3 CA1 PrL BLA aRSC ACC alC

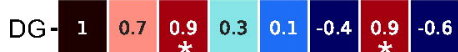

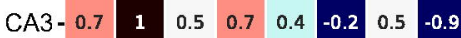
\begin{tabular}{lllllll|l|l} 
CA1 $1-0.9$ & 0.5 & 1 & 0.4 & 0.3 & -0.8 & 1 & -0.3 \\
$*$
\end{tabular} $\begin{array}{ccccccccc}\text { PrL- } 0.3 & 0.7 & 0.4 & 1 & 0.9 & -0.8 & 0.4 & -0.5\end{array}$ \begin{tabular}{llll|l|l|l|l} 
BLA - 0.1 & 0.4 & 0.3 & 0.9 & 1 & -0.8 & 0.3 & -0.3
\end{tabular} \begin{tabular}{ll|l|l|l|l|l|l|lllllll|l|l|l|l}
\hline aRSC -0.4 & -0.2 & -0.8 & -0.8 & -0.8 & 1 & -0.8 & 0.2 & aRSC -0.6 & 0.46 & 0.6 & 0.9 & 1 & 1 & 0.9 & 0.9 \\
$\star$ & $*$ &
\end{tabular} \begin{tabular}{lllllll|l|l} 
ACC -0.9 & 0.5 & 1 & 0.4 & 0.3 & -0.8 & 1 & -0.3 \\
\hline & $\star$ & -1
\end{tabular}

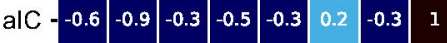
D'G CÁ3 CÁ1 PrLBLÁ aRŚ ÁCC alC
$0.3 \mathrm{~mA}$

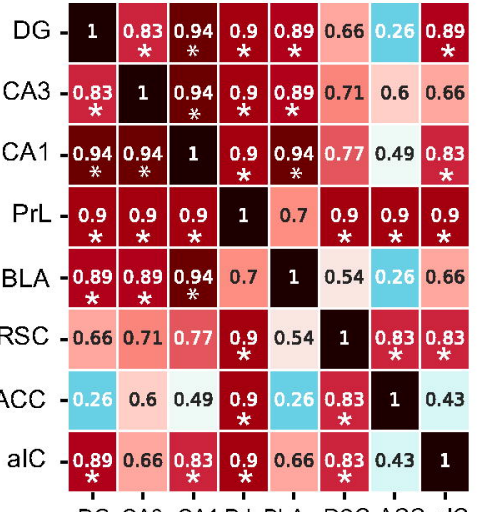

\begin{tabular}{l|c|c|c|c|c|c|c|}
\hline DG - 1 & 0.97 & $\begin{array}{l}1 \\
*\end{array}$ & 0.7 & 0.6 & 0.6 & 0.7 & 0.8 \\
\hline
\end{tabular} \begin{tabular}{l|l|l|llllll} 
CA3 -0.97 & 1 & 0.97 & 0.62 & 0.46 & 0.46 & 0.62 & 0.72
\end{tabular}

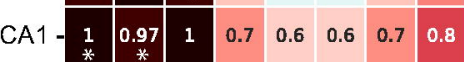
\begin{tabular}{ll|l|l|l|l|l|l} 
PrL - 0.7 & 0.62 & 0.7 & 1 & 0.9 & 0.9 & $\frac{1}{*}$ & 0.8
\end{tabular} \begin{tabular}{llll|l|l|l|l|l} 
BLA - 0.6 & 0.46 & 0.6 & 0.9 & 1 & 1 & 0.9 & 0.9
\end{tabular} \begin{tabular}{ll|l|l|l|l|l|l|l} 
ACC -0.7 & 0.62 & 0.7 & $\underset{*}{1}$ & $\begin{array}{c}0.9 \\
\star\end{array}$ & $\begin{array}{c}0.9 \\
\star\end{array}$ & 1 & 0.8 \\
\hline
\end{tabular} \begin{tabular}{l|l|l|l|l|l|l|l} 
alC - 0.8 & 0.72 & 0.8 & 0.8 & 0.9 & 0.9 & 0.8 & 1 \\
\hline
\end{tabular} D'G CÁ3 CÁ1 PrLBLÁA aRŚC A'CC á́C

\section{$1.0 \mathrm{~mA}$}
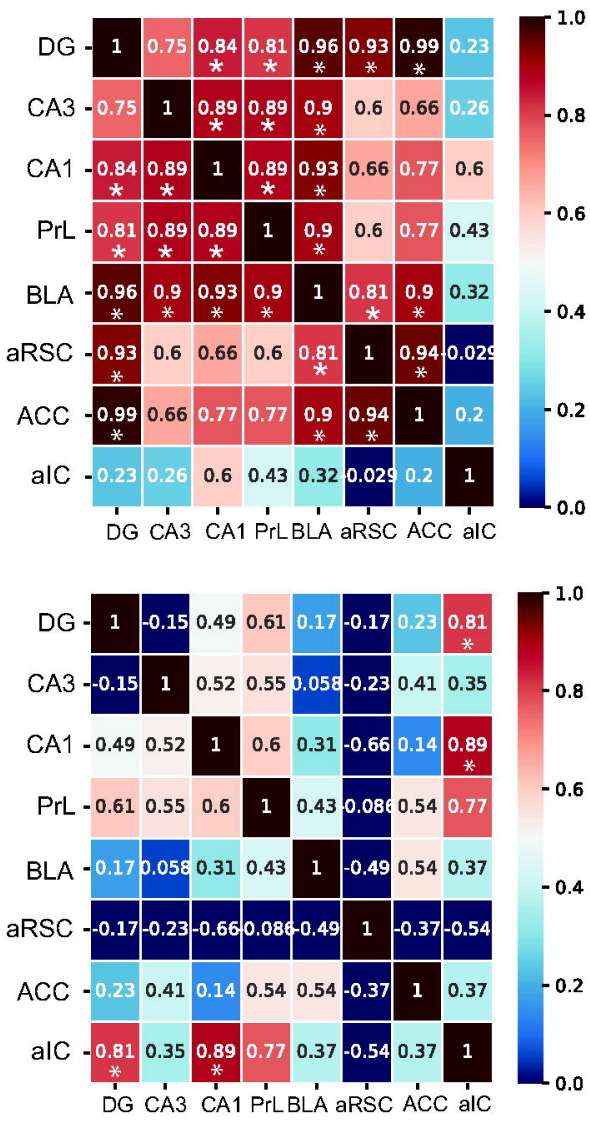

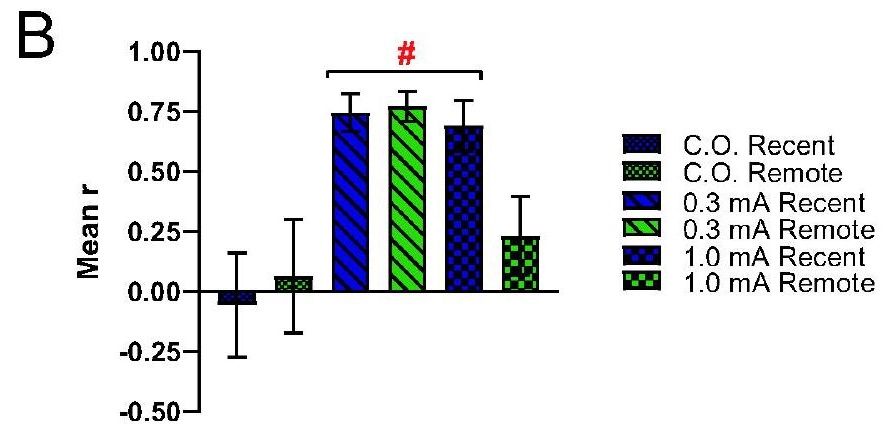

